\title{
Acceleration-based Transparency Control Framework for Wearable Robots
}

\section{Conference Paper}

Author(s):

Boaventura, Thiago; Buchli, Jonas

Publication date:

2016

Permanent link:

https://doi.org/10.3929/ethz-a-010736126

Rights / license:

In Copyright - Non-Commercial Use Permitted 


\title{
Acceleration-based Transparency Control Framework for Wearable Robots
}

\author{
Thiago Boaventura, Jonas Buchli
}

\begin{abstract}
To render a wearable robot imperceptible to a user is a very challenging control task. The constant and intrinsic interaction between robot and human, and persondependent behaviours are the main difficulties when designing such cooperative control. In this contribution we introduce and discuss a novel and promising transparency control framework. The foundation of the framework is to measure the acceleration of the human limbs and to exploit this measurement to generate feedforward control commands by using a rigid body model of the robot. The framework includes also an acceleration feedback controller and a state estimator to enhance the overall performance. We present a simplified stability analysis with different feedback controllers and preliminary experimental data that demonstrate the potential of the proposed method in reducing interaction forces and mimicking human motions.
\end{abstract}

\section{INTRODUCTION}

To improve human quality of life is a major goal in the field of wearable robotics, which includes e.g. exoskeletons and limb-prostheses. These devices are closely attached to the human body in a symbiotic way, moving and working in tandem with the human. They can be employed in a wide range of applications such as physical assistance, rehabilitation, and power augmentation. In this work, we focus on exoskeletons for healthy users, where the robot should not apply corrective forces to the user nor impose a gait pattern, but only follow the user motions in a natural way.

In this contribution we will present a novel control framework to enhance the transparent behaviour of wearable robots. At the core of our approach lies the idea to mimic the human motions with as low lag as possible and, therefore, minimise the human-robot interaction forces. To do so, we measure and use the acceleration of the human limbs to produce feedforward control inputs for the robot. These commands are generated using the robot floating base rigid body model, which allows to compute the required torques needed to reproduce the human motions based on the acceleration of the limbs of the wearer. This model-based control approach has been successfully employed in several robotics platforms e.g. [1], [2], but to the best of our knowledge its application to wearable robots has not yet been shown.

Besides the essential feedforward model-based command mentioned above, our novel transparency control framework includes an acceleration feedback controller to reassure human and robot move with the same acceleration and speed, and a state estimator to drive the remaining interaction forces to zero. In short, the main contributions of this paper are: 1) a novel transparency control framework, which does not require any a priori human model, and may use only inertial

T.Boaventura and J. Buchli are with the Agile \& Dexterous Robotics Lab, ETH Zurich. tboventuradethz.ch, buchlijeethz.ch measurement units (IMUs) and the floating base rigid body model of the robot to compensate for inertial and gravitational forces; 2) a stability analysis of the proposed method; and 3) preliminary experimental results that demonstrate the potential of the proposed algorithm.

Last but not least, although the focus of our work is on wearable robots, this framework can well be used in different applications in which it is desirable to reproduce a human motion with minimum lag, such as haptics and virtual reality.

\section{STATE OF THE ART REVIEW}

Wearable robots, such as exoskeletons, are nowadays a strong and well-established research topic worldwide, and in this section we will mention some of the most relevant research outcomes in this field related to our work. The state of the art in transparency control currently relies on interaction force feedback, impedance and admittance controllers, and/or electromyography (EMG).

Probably the most common way of reducing the humanrobot interaction forces is by actively reducing the apparent robot impedance at its contact points with the human. With impedance control it is possible to set a desired dynamic behaviour for the robot interaction ports. However, values for desired stiffness, damping, and inertia cannot be arbitrarily low. There are fundamental limitations on the stability of such controllers and on the amount of inertia it can mask (i.e. hide). Colgate and Hogan have shown in [3] that, given a uniform robot model, a feedback force controller can mask maximally half of the original mass. They also mention that this theoretical limitation is rather optimistic and that in practice stability limitations may be even more severe.

In some cases, a wearable robot does not guarantee stability properties intentionally. In [4], an admittance controller is used to mask the dynamics of a 1-DOF articulated leg from the user. In addition to the admittance controller, a positive feedback of the exoskeleton joint acceleration is used to compensate inertia. The inertia is slightly over compensated, and the system is unstable in isolation. It is the human that provides the stabilising dynamics.

An alternative to overcome fundamental stability limits on force feedback control and on its ability to mask inertia is to employ model-based feedforward controllers. Common and intuitive model-based controllers include gravity and friction compensation, which only require models from the robot and not from the human. For instance, [5] uses such compensations in addition to an interaction force feedback control. In [6], besides joint torque feedback controllers, a feedforward command that realises a conservative force field is proposed. Although the proposed algorithm does not need 


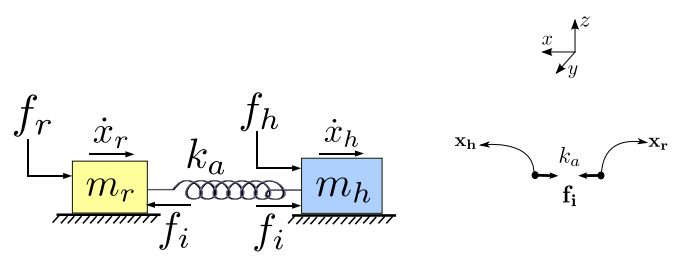

Fig. 1. Conceptual linear 1 DOF representation of a robot (link) attached to a human (link), as well as a schematic of a possible real human-robot attachment. The robot and human have inertias $m_{r}$ and $m_{h}$, and velocities $\dot{x}_{r}$ and $\dot{x}_{h}$ respectively. The attachment is modelled as a spring with stiffness $k_{a}$, which creates the interaction forces $f_{i}$. The human force $f_{h}$ comes from muscle contraction, and the robot can use its actuation system to apply the force $f_{r}$ to its links.

a human model, it requires the a priori knowledge of user's preferred motions. In [7], 3 different models of the dynamics of the exoskeleton for distinct gait phases, as well as a human muscle model, are used to design a low-impedance (i.e. high-sensitivity) feedback controller for BLEEX. A different control approach is employed in the exoskeleton HAL, which uses EMGs to detect the user intentions and then assist the user in tasks such as walking and load carrying [8].

A possible alternative to improve transparency controller is by using bio and neuromechanical models that are able to qualitatively describe human motions e.g. walking [9], [10]. Given the model is accurate, the exoskeleton could replicate the expected human motions to possibly increase its imperceptibility. However, such models are not yet able to satisfactorily describe a wide range of human tasks and often create only conservative results. To improve such models optimal and learning control algorithms can be used [11].

Last but not least, although the use of IMUs on wearable robots is not novel, their use has been restricted mainly to the detection of gait initiation and termination, and as input to state estimators that aim at retrieving kinematic information [12]. Their effective use on control loops has also been limited. For instance, in [13] IMUs were used to implement a damping control to reduce user's tremors in an upper limb exoskeleton. Thus, to the best of our knowledge, the use of IMUs to enhance transparency in wearable robots is a novel application, and we believe their use on real-time control has an enormous potential. Such sensors are rapidly improving their signal quality and latencies, and also becoming more affordable. Finally, IMUs are lightweight and generally easy to be integrated on the robot hardware.

\section{Conceptual Model}

A wearable robot is always connected to the wearer via a compliant attachment. To obtain a better understanding on the human-robot interaction dynamics, we will first derive a simple and conceptual 1 degree of freedom linear model that is still able to capture the essence of such coupled system.

\section{A. Modelling}

Considering both human and robot links as simple inertias, and the attachment as a purely elastic element, we can draw the scheme depicted in Fig. 1. The force $f_{h}$ characterise any force profile applied by the human to its own body, while $f_{r}$ represents the forces exerted by the wearable robot. Given a relative motion between robot and human, the attachment stiffness $k_{a}$ defines the dynamics of the interaction force $f_{i}$. The dynamics of the coupled system can be described through the three following equations:

$$
\begin{gathered}
\dot{f}_{i}=k_{a}\left(\dot{x}_{r}-\dot{x}_{h}\right) \\
f_{r}-f_{i}=m_{r} \ddot{x}_{r} \\
f_{h}+f_{i}=m_{h} \ddot{x}_{h}
\end{gathered}
$$

These simple equations can be combined in different ways. Given our control input is $f_{r}$ and desired output is $f_{i}$, we can rewrite the interaction force dynamics as:

$$
m_{r} \ddot{f}_{i}+k_{a} f_{i}=k_{a}\left(f_{r}-m_{r} \ddot{x}_{h}\right)
$$

Since our overall goal in this paper is to make a wearable robot imperceptible to the user, we should control the dynamics of the interaction force $f_{i}$ such that it converges as quickly as possible to zero.

\section{B. Control Approaches}

There are different possibilities on how to control the human-robot interaction forces in order to make the wearable robot transparent to the wearer. Usually either a low impedance control or a feedback control loop on the interaction force is employed. In this section we will show that by measuring the human acceleration both model-based feedforward control and acceleration feedback control proposed by our framework can be used to enhance the interaction force control. All the approaches described in this section consider that the robot is force-controlled and able to provide the desired force $f_{r}$ (or torques) calculated by the controller.

1) Model-based feedforward control: As we can see in Eq. 4, the term $m_{r} \ddot{x}_{h}$ in the right hand side depends both on the human acceleration and on the robot inertia. This term can be seen as a human disturbance input to the interaction force dynamics. No matter if and what feedback controller is used, to a priori compensate for this human influence will always improve the interaction force control performance. Also, by compensating for this term, the interaction force dynamics becomes human-independent, which is a very attractive feature that boosts the versatility of the controller. To compensate for this term, a controller should at all times provide a feedforward force $f_{r_{f f}}$ as follows:

$$
f_{r_{f f}}=m_{r} \ddot{x}_{h}
$$

Equation 5 inverts the robot forward dynamics to produce a force command that compensates for the human acceleration influence in the loop. This means the calculation of $f_{r_{f f}}$ requires knowledge of the robot dynamics model (in this simple linear case only the mass $m_{r}$ ) and also the measurement of the human acceleration $\ddot{x}_{h}$.

Given $f_{r_{f f}}$ perfectly cancels out the term $m_{r} \ddot{x}_{h}$ in Eq. 4 , the roots of the interaction force characteristic equation would be equal to $s= \pm \sqrt{-k_{a} / m_{r}}$, which means the system response would be purely oscillatory, where the frequency of the oscillations is defined by the attachment 
stiffness and the robot inertia. Clearly, this is not a sufficient controller on its own since it is not able to set the force null. To do so we need, additionally, a feedback controller. Thus, the following sections will introduce two possible feedback controllers that are able to set a convergent dynamics for the interaction force dynamics.

2) Interaction force feedback control: To reach transparency, the most intuitive choice is to measure and control the quantity we want to regulate, i.e. the interaction force $f_{i}$. However, to instrument the attachment interface with force sensors is usually not easy, and often avoided. Yet, in case a measurement of $f_{i}$ is available, a feedback e.g. $\mathrm{PD}$ (proportional-derivative) controller can be added to the feedforward command as follows:

$$
f_{r}=f_{r_{f f}}+k_{p}\left(f_{i_{d}}-f_{i}\right)+k_{d}\left(\dot{f}_{i_{d}}-\dot{f}_{i}\right)
$$

where $f_{i_{d}}$ is the desired interaction force, and $k_{p}$ and $k_{d}$ are the proportional and derivative gain respectively. To reach transparency we set $f_{i_{d}}$ and $\dot{f}_{i_{d}}$ as zero, and the roots of the characteristic equation are be given by $s=\frac{-k_{a} k_{d} \pm \sqrt{\left(k_{a} k_{d}\right)^{2}-4 m_{r}\left(1+k_{p}\right)}}{2 m_{r}}$. The controller gain $k_{p}$ can be designed, for instance, to produce a critically-damped response, and $k_{d}$ to satisfy a desired settling time.

3) Acceleration feedback control: To avoid the additional complexity of adding a force sensor at the attachment interface, a feedback loop on the acceleration instead of on the interaction force could be closed. To do so, additionally to the human acceleration, used in the feedforward command $f_{r_{f f}}$, we would need to measure the robot acceleration as well. Although it is possible to obtain the acceleration signal from double differentiation of the position signal, this in practice usually results in a poor measurement either because of noise amplification or lag due to filtering. Thus, we suggest the use of a second IMU on the robot to directly measure its acceleration. An IMU can be attached simply on the robot structure, making its mechanical integration much simpler than the one of a load cell. By measuring both human and robot accelerations, an acceleration PI feedback controller can be designed, where the human acceleration would be the desired acceleration for the robot:

$$
f_{r}=f_{r_{f f}}+k_{p}\left(\ddot{x}_{h}-\ddot{x}_{r}\right)+k_{i}\left(\dot{x}_{h}-\dot{x}_{r}\right)
$$

where $k_{p}$ and $k_{i}$ are the proportional and integral gains. With such control law, it is possible to obtain an interaction force dynamics similar to the one obtained by directly feeding back $f_{i}$ as described in Sec. III-B.2. That is, the roots of the characteristic equation in this case would be given by $s=\frac{-k_{i} \pm \sqrt{k_{i}^{2}-4\left(m_{r}+k_{p}\right) k_{a}}}{2\left(m_{r}+k_{p}\right)}$, where the gains $k_{p}$ and $k_{i}$ can be designed to produce a desired convergent dynamics for the interaction force.

\section{Multi-DOF ARTiCulated CASE}

In Sec. III we analysed the transparency control of a simple linear 1 DOF conceptual model of a coupled humanrobot system, and obtained important insights, such as:

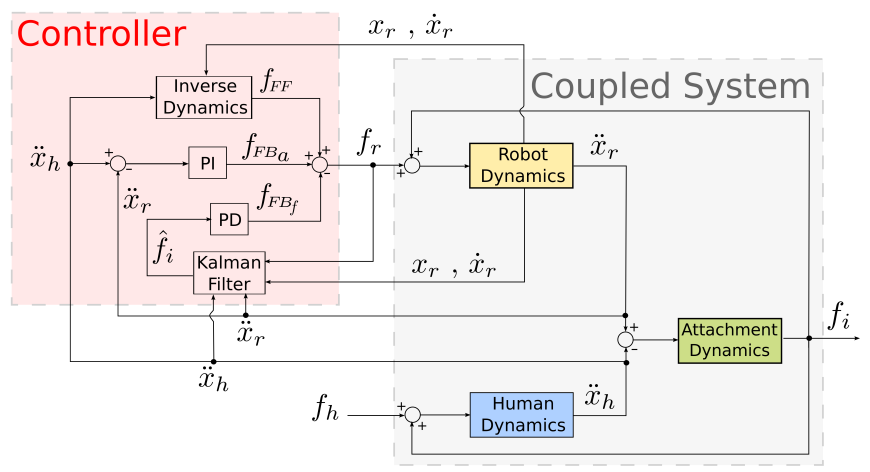

Fig. 2. Complete transparency control framework. It consists of 3 main blocks: 1) a feedforward inverse dynamics block, which uses state feedback from the robot and the human acceleration $\ddot{x}_{h} ; 2$ ) an acceleration feedback controller (e.g. a PI), in which the human acceleration $\ddot{x}_{h}$ is the target value for the robot acceleration $\ddot{x}_{r}$; and 3) a state estimator (e.g. Kalman filter) for the interaction force followed by a feedback controller (e.g. a PD), being the desired interaction force value zero.

- A model-based feedforward command using the human acceleration as input is able to compensate for the human influence in the interaction force dynamics;

- An acceleration feedback control is able to produce the same interaction force dynamics in closed-loop as when the interaction force itself is fed back, with the advantage that its practical implementation is simpler.

In the rest of this section, we will show that these points are also valid for more complex system, such as articulated and multi-DOF robotic devices and human models.

\section{A. Modelling}

Traditional robots can be well modelled as a rigid body system. Since such robots are not fixed to their environment, they are called floating base robots. The floating base rigid body dynamics for the coupled human-robot system can be described as following:

$$
\mathbf{M}(\mathbf{q}) \ddot{\mathbf{q}}+\mathbf{h}(\mathbf{q}, \dot{\mathbf{q}})=\mathbf{S}^{T} \tau+\mathbf{J}_{\mathbf{c}}{ }^{T} \lambda+\mathbf{J}_{\mathbf{a}}{ }^{T} \mathbf{f}_{i}
$$

where $\mathbf{q}$ is the vector of robot joint positions; $\mathbf{M}(\mathbf{q})$ is the robot floating base inertia matrix; $\mathbf{h}(\mathbf{q}, \dot{\mathbf{q}})$ the centripetal, Coriolis, and gravity forces vector; $\mathbf{S}^{T}$ is the robot actuated joint selection matrix; $\tau$ the robot torque command vector; $\mathbf{J}_{\mathbf{c}}$ is the Jacobian matrix of the linearly independent constraints forces $\lambda ; \mathbf{J}_{\mathbf{a}}$ is the Jacobian matrix of the attachment point $\mathbf{x}_{\mathbf{r}}$ (see Fig. 1); and $\mathbf{f}_{i}$ the human-robot interaction force. ${ }^{1}$ More details on the modelling and control of floating base robots may be found in [2].

The interaction force vector can be defined as $\mathbf{f}_{i}=$ $\left(f_{i_{x}}, f_{i_{y}}, f_{i_{z}}\right)^{T}$, which includes the components of the force in the inertial frame and can be defined as:

$$
\dot{\mathbf{f}}_{\mathbf{i}}=k_{a}\left(\dot{\mathbf{x}}_{\mathbf{r}}-\dot{\mathbf{x}}_{\mathbf{h}}\right)
$$

where $\mathbf{x}=(x, y, z)$ is the position in the inertial coordinate system for both robot and human (Fig. 1).

\footnotetext{
1 As for the mathematical notation, matrices are in bold-face capital letters; vectors are bold-face and lower-case; while scalars are also lowercase but not in bold-face.
} 


\section{B. Control}

In this section, we will only extend the feedforward modelbased controller to the multi-DOF articulated case. The feedback analysis is similar to the 1-DOF case, and it will not be included in this paper due to space limitations.

1) Floating base rigid body inverse dynamics feedforward control: As we discussed in Sec. III-B.1, the feedforward command that tries to mimic the human acceleration requires the inversion of the robot dynamics. In the multi-DOF articulated case, the robot dynamics is given by the floating base rigid body dynamics described in Eq. 8. The inverse of this model provides us the joint torques $\tau$ required to create a desired joint angular acceleration $\ddot{\mathbf{q}}_{\mathbf{d}}$, compensating also for the Coriolis and gravitational forces.

Since our goal is to follow the human motions to enhance transparency, the desired robot angular accelerations in our case are set as the human angular accelerations. To convert the robot and human IMU acceleration readings to joint angular accelerations, a Jacobian matrix can be defined given the location of the IMUs are known. The most appropriate location of the IMUs on the robot and human links will depend on several factors, such as number of attachment points, robot kinematics, etc. This issue is discussed in more details on Sec. VII.

To overcome the intrinsic complexity related to floatingbase robots such as under-actuation, dynamically changing contact states, and contact forces that may not be known, an orthogonal decomposition can be used to calculate the floating base inverse dynamics. Details are given in [2] and will not be reported in this paper due to space constraints.

\section{TRANSPARENCY CONTROL FRAMEWORK}

The control law described in Eq. 7 aims at making the robot move at the same acceleration and speed as the human. However, it is intuitive to see that even when the robot moves with exactly the same speed and acceleration of the human, one cannot assure the value of the interaction force since it will depend on the relative position of both robot and human, that is, on how much the attachment element is compressed/extended at that time. In other words, although the interaction force dynamics (time derivatives of $f_{i}$ ) converges to zero, it does not mean the interaction force is null. Thus, to further enhance the transparency control framework, we propose the use of a state estimator (e.g. a Kalman filter) to close a feedback loop on the estimated interaction forces. Of course, in case a force sensor is available or preferred, the direct interaction force measurement could be used instead of its estimation.

This state estimator could use different inputs for estimating the interaction force. Besides the human and robot accelerations, measured e.g. with IMUs, the estimator could also use the robot position and velocities. Such measurements, usually obtained by joint high-resolution encoders, are normally available in any robot hardware. To further enhance the estimator performance, any additional knowledge that may be beneficial could also be used, such as the compliant characteristics of the attachment element, as well as human models or trajectories data.

The final transparency control framework we propose is depicted in Fig. 2, and includes a feedforwad inverse dynamics block, an acceleration feedback block, and an interaction force estimator. We highlight the implementation of the entire framework is beyond the scope of this paper, which focuses instead on presenting the framework, and demonstrating the capabilities of its core part: the modelbased feedforward command described in Eq. 5.

\section{PReliminary Results}

In this section we will present some preliminary experimental results that were designed as a proof of concept.

\section{A. Linear testing rig}

To start validating our control framework, we have designed and built a linear 2 DOF testing rig, which exactly emulates the conceptual model depicted in Fig. 1. In this rig, the robot and human masses are $m_{r}=m_{h}=3.6 \mathrm{~kg}$, the spring attachment stiffness is $k_{a}=4.75 \mathrm{kN} / \mathrm{m}$, and the interaction force $f_{i}$ was estimated through the deflection of the linear spring that connects both masses by using highresolution encoders that measure their positions.

With this platform, we performed an experiment to demonstrate the impact of the model-based feedforward part of the proposed transparency framework in the interaction force dynamics. In this experiment, we have set a $3 \mathrm{~Hz}$ sinusoidal position trajectory for the "human", while the "robot" was programmed with two different controllers: during the initial $1.5 \mathrm{~s}$, a pure "zero impedance" feedback controller, where a constant zero force reference was sent to the "robot" low level controller; and afterwards, the model-based feedforward controller of Eq. 5, where the "human" acceleration was measured with an IMU and the "robot" desired force was set as $f_{r_{f f}}$. As we can see in the second plot of Fig. 3, after a transient period the range of the interaction force $f_{i}$ decreases from $[-45 ; 40] \mathrm{N}$ to $[-9 ; 5] \mathrm{N}$ when the acceleration-based feedforward force command is switched on. This represents a significant reduction of more than 6 times in the total interaction force amplitude, demonstrating the potential of this core component of the proposed framework.

\section{B. HyQ leg}

In this section we present the results of an experiment with one leg of the quadruped robot HyQ. This complements the previous result by showing the performance of the algorithm for an articulated robot. In this case, because HyQ is not a wearable robot, safety issues raised and therefore the human was not attached to the robot. Thus, in this experiment there is no interaction force at all between human and robot. Instead of looking at the interaction force dynamics, the goal in here was to purely observe the capabilities of the feedforward controller in isolation (i.e. with no influence of the human and attachment dynamics) to mimic the human motions in real time. 


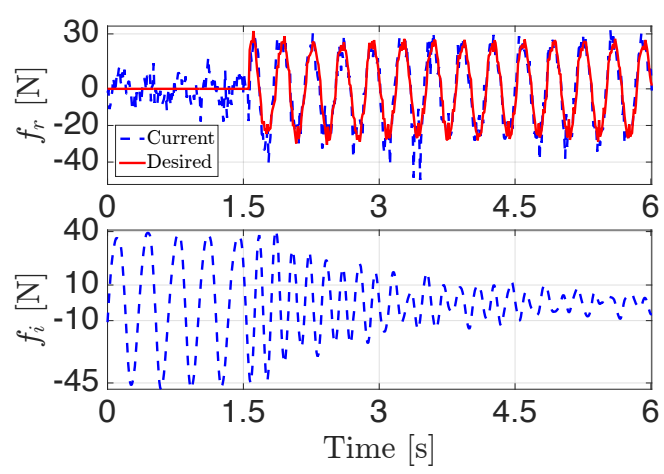

Fig. 3. Interaction force dynamics with two different controllers: till $1.5 \mathrm{~s}$ a zero force feedback controller was used, and afterwards the accelerationbased feedforward command (Eq. 5) was applied. As we can see in the first plot, the "robot" is able to satisfactorily track the desired forces generated based on the "human" acceleration measurement. The use of such feedforward command reduced the interaction force amplitude in more than 6 times with respect to the "zero impedance" controller.
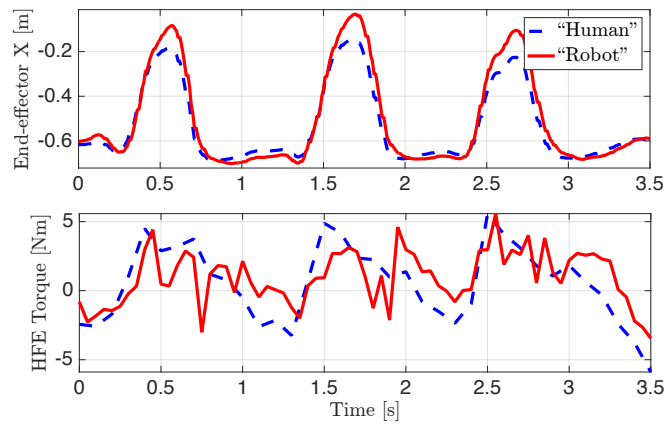

Fig. 4. The first plot shows the end-effector position tracking for the $x$ direction. The dashed blue line depicts the human position reference, obtained from double integrating the IMU acceleration signal, and the solid red line the real motion performed by the robot leg. As we can see, the feedforward floating base inverse dynamics controller alone is able to follow the human motions with essentially no delay. The second plot shows in dashed blue the desired feedforward torques, and in solid red the real torques applied by the HFE joint.

The experiment consisted of having the human holding an IMU in the hand and moving the forearm up and down by moving only the elbow joint (shoulder joint did not move). Then, we set the IMU accelerations from the human hand as desired end-effector accelerations $\ddot{x}_{\mathbf{e e}_{\mathbf{d}}}$ for the robot. These end-effector accelerations were then mapped into joint space desired accelerations $\ddot{q}_{d}$ by using the appropriate leg Jacobian $\mathbf{J}_{\text {leg }}$ and the dynamic relation $\ddot{\mathbf{q}}_{\mathbf{d}}=\mathbf{J}_{\text {leg }}{ }^{-1}\left(\ddot{\mathbf{x}}_{\mathbf{e e}_{\mathbf{d}}}-\right.$ $\left.\dot{\mathbf{J}}_{\text {leg }} \dot{\mathbf{q}}\right)$. Finally, the desired accelerations $\ddot{\mathbf{q}}_{\mathbf{d}}$ were fed into the inverse dynamics algorithm, which calculated the feedforward joint torques required to mimic the human hand motion.

In Fig. 4 we show both the human and robot end-effectors position in the $x$ direction, and the respective torque tracking for the hip flexion-extension (HFE) joint of the leg. We can see in the first plot of Fig. 4 that, even with no feedback loop and despite an overshoot, the human motion could be qualitatively followed with practically no delay. Since the system was uncoupled, we can affirm that the robot motion was entirely produced by the acceleration-based feedforward command, with no help of the human at all.
The non-perfect torque tracking, showed in the second plot, as well as inaccuracies in the leg rigid body model and sensor/processing delays are some of the aspects that limit the human motion tracking capabilities in this case.

\section{DISCUSSION}

Our work aims at providing transparency to wearable robots, such as exoskeletons, during daily activities, including very dynamic manoeuvres such as running. We would like the user to perform such motions as if the robot was not even attached to him/her. To reach such levels of transparency, it is paramount to reduce the apparent inertia of the robot (i.e. the inertia the user feels) and not only compensate for gravitational and friction forces.

One way to reduce the inertia of the exoskeleton is by hardware, where the robot links are made as light as possible. Because of the power required to perform dynamic motions, and potentially to help the user carrying external loads, the robot structure needs some mechanical robustness, creating a trade-off between power and weight. An alternative to further reduce the apparent inertia though hardware is by using series elastic actuators [14], [15].

Besides the importance of building an adequate hardware, it is through control software that transparency is mainly achieved. In order to reduce the apparent robot inertia (or more generally speaking its impedance) at the interaction port, a force/torque feedback controller should be used. The approach we described in this paper relies on high-fidelity low-level torque controllers.

To enhance the performance of pure force feedback controller in reaching transparency, we propose the use of a feedforward force/torque command that requires two main elements: the rigid body model of the robot, along with the measurement of its states; and the measurement of the human accelerations. Since the approach is model-based, it is clear that the more accurate the robot model, the better the human acceleration tracking. Furthermore, the human accelerations should be measured and filtered with as low latency as possible to minimise the lag between human and robot motions. Also, since the inverse dynamics controller is a marginally stable controller, an additional control element has to be added to avoid drift and offsets due to modelling and measurement errors and sensors noise. The physical connection between the human and the exoskeleton or a nonzero gain for the position feedback controller could provide such stabilising dynamics on a full-body robot.

The approach we can choose to control the humanrobot interaction forces depends mainly on the sensors we have available. The best scenario is the one where we could measure everything, i.e. the interaction force itself, the human acceleration, and the human muscles activation. However, this scenario is not realistic in most of the cases. To measure the interaction force a single or multiple-axes load cell should be added to the interface between the robotic link and the user, increasing costs and design complexity. On the other hand, to obtain an estimation of the human forces, electromyographs (EMGs) should be employed. However, 
the use of this technology is very time consuming and often impractical. Finally, the measurement of the human acceleration requires the use of IMUs. A benefit of using an IMU attached to the human is that it would minimally impact the robot design. IMUs' increasing commercial availability are making such technology more affordable and their quality is constantly improving, with latencies suitable for real time control [16]. In this work we have used the XSens MTi 100 IMU [17]. We highlight that the availability of interaction force sensors or EMGs do not preclude, but complement the proposed framework.

The most appropriate location of the IMU on the human depends on several factors and it is also a design decision. In principle, all the human links that are attached to the exoskeleton should have an IMU, which could be placed for instance at the attachment cuffs. In case there is no redundancy on the robot kinematic structure, a single IMU could be placed at the end effector instead of at the joints. Anyhow, independently of where the IMU is placed, in case the human kinematics is known it is possible to define a Jacobian matrix that maps the acceleration from the IMU space to the human joint space.

Last but not least, although the focus of this paper is on wearable robots for healthy subjects, the framework can also be used for rehabilitation purposes. For instance, with such transparency controller it is possible to provide assistance as needed, that is, to set some joints passive (i.e. transparent) while others are active, permitting also the training of single joints at a time. Furthermore, this approach can be very beneficial also in other fields where it is desirable to reproduce a human motion with minimum lag such as haptics, virtual reality, and gaming.

\section{CONCLUSIONS AND FUTURE WORK}

To reach transparency with a wearable robot means to guarantee zero interaction force and lag between the robot and the user. This is a very challenging task, since human actions can be very unpredictable and significantly vary among different subjects. Traditional control methods include impedance control and interaction force feedback control, however they have fundamental limitations and may considerably increase the robot design complexity. We presented a novel control method which is capable of enhancing the transparency performance and yet is easy to be integrated on the robot design. It consists of measuring the human acceleration though IMUs, and using this signal as input to a model-based feedforward controller. In addition, our framework does not require a human model, although it could definitely benefit from such a model.

To ensure stability, this feedforward controller should be used together with a feedback controller. We have presented simple stability analyses that show that an acceleration feedback controller is able to produce the same system response as an interaction force feedback controller. Then we presented preliminary experimental results that, not aiming at setting a benchmark, only demonstrated the potential of the feedforward command to mimic human motions and to reduce the interaction force amplitude.

The work presented in this paper is an important step towards an enhanced transparency controller for wearable robots. Future work includes investigating the influence of the stiffness of the human-robot attachment on the control performance and robustness; and ultimately the implementation of the full framework in an exoskeleton robot.

\section{ACKNOWLEDGEMENTS}

The authors would like to thank the support of XSens. This research has been supported by a Swiss National Science Foundation Professorship Award to Jonas Buchli and the FP7 EU Project BALANCE (Grant 601003).

\section{REFERENCES}

[1] T. Boaventura, C. Semini, J. Buchli, M. Frigerio, M. Focchi, and D. G. Caldwell, "Dynamic torque control of a hydraulic quadruped robot," in IEEE International Conference in Robotics and Automation (ICRA), 2012, pp. 1889-1894.

[2] M. Mistry, J. Buchli, and S. Schaal, "Inverse dynamics control of floating base systems using orthogonal decomposition," IEEE Int. Conf. on Robotics and Automation (ICRA), pp. 3406-3412, 2010.

[3] E. Colgate and N. Hogan, "The interaction of robots with passive environments: Application to force feedback control," in Advanced Robotics: 1989. Springer, 1989, pp. 465-474.

[4] G. Aguirre-Ollinger, J. Colgate, M. Peshkin, and A. Goswami, "Inertia compensation control of a one-degree-of-freedom exoskeleton for lower-limb assistance: Initial experiments," Neural Systems and Rehabilitation Engineering, IEEE Transactions on, vol. 20, no. 1, pp. 68-77, Jan 2012.

[5] D. Zanotto, T. Lenzi, P. Stegall, and S. Agrawal, "Improving transparency of powered exoskeletons using force/torque sensors on the supporting cuffs," in Rehabilitation Robotics (ICORR), 2013 IEEE International Conference on, June 2013, pp. 1-6.

[6] H. Vallery, A. Duschau-Wicke, and R. Riener, "Optimized passive dynamics improve transparency of haptic devices," in IEEE Int. Conf. on Robotics and Automation (ICRA), 2009, pp. 301-306.

[7] H. Kazerooni and R. Steger, "The berkeley lower extremity exoskeleton," ASME Journal of Dynamic Systems, Measurement and Control, vol. 128, pp. 14-25, 2006.

[8] H. Kawamoto and Y. Sankai, "Power assist method based on phase sequence and muscle force condition for HAL," Advanced Robotics, vol. 19 , no. 7, pp. 717-734, 2005.

[9] R. Blickhan, "The spring-mass model for running and hopping," Journal of Biomechanics, vol. 22, no. 11-12, pp. 1217-1227, 1989.

[10] H. Geyer and H. Herr, "A muscle-reflex model that encodes principles of legged mechanics produces human walking dynamics and muscle activities," IEEE Trans Neural Syst Rehabil Eng, vol. 18, no. 3, pp. 263-273, 2010.

[11] H. Vallery, A. Duschau-Wicke, and R. Riener, "Generalized elasticities improve patient-cooperative control of rehabilitation robots," in IEEE Int. Conf. on Rehabilitation Robotics (ICORR), 2009, pp. 535-541.

[12] D. Novak, P. Reberšek, S. M. M. De Rossi, M. Donati, J. Podobnik, T. Beravs, T. Lenzi, N. Vitiello, M. C. Carrozza, and M. Munih, "Automated detection of gait initiation and termination using wearable sensors," Medical engineering \& physics, vol. 35, no. 12, pp. 1713$1720,2013$.

[13] E. Rocon, J. Moreno, A. Ruiz, F. Brunetti, J. Miranda, and J. Pons, "Application of inertial sensors in rehabilitation robotics," in $R e$ habilitation Robotics, 2007. ICORR 2007. IEEE 10th International Conference on. IEEE, 2007, pp. 145-150.

[14] G. Pratt and M. Williamson, "Series elastic actuators," in IEEE Int. Conf. on Intelligent Robots and Systems (IROS), 1995.

[15] J. Pratt, B. Krupp, and C. Morse, "Series elastic actuators for high fidelity force control." Industrial Robot, vol. 29, no. 3, pp. 234-241, 2002.

[16] H.-P. Bruckner, W. Theimer, and H. Blume, "Real-time low latency movement sonification in stroke rehabilitation based on a mobile platform," in Consumer Electronics (ICCE), 2014 IEEE International Conference on, Jan 2014, pp. 264-265.

[17] A. Vydhyanathan, G. Bellusci, H. Luinge, and P. Slycke, "The next generation xsens motion trackers for industrial applications," 2014. 